the procedure and how to take BP. A randomised study comparing patients with access to a video versus no access would confirm the benefit of standard use of this educational tool for patients.

\section{PTH-062 ENDOSCOPIC MANAGEMENT OF BURIED BUMPER SYNDROME (BBS) USING A DEDICATED RESECTION DEVICE: THE 'FLAMINGO SET'}

Deborah Costa, Edward J Despott, Nikolaos Lazaridis*, Nikolaos Koukias, Andrea Telese, Claudia Coppo, Alberto Murino. Royal Free Unit for Endoscopy, The Royal Free Hospital and University College London (UCL) Institute for Liver and Digestive Health, London, UK

\subsection{6/gutjnl-2019-BSGAbstracts.87}

Introduction Buried bumper syndrome (BBS) is an uncommon complication of percutaneous endoscopic gastrostomy (PEG) placement, with an incidence of $1 \%$. Several techniques for endoscopic management of BBS have been described, given the absence of a dedicated device to date.

Methods A 94-year-old man presented with fever and PEG obstruction. A PEG had been placed in 2014 for enteral feeding in the context of dysphagia, secondary to Parkinson's disease. On examination, the cutaneous side of the PEG tract appeared erythematous and oedematous, with seepage of purulent mucus; any attempt to mobilise the PEG tube though external manipulation proved futile.

Results At upper gastrointestinal (GI) endoscopy, a $4 \mathrm{~cm}$ elevated area of granulomatous tissue with a central depression was identified on the proximal anterior wall of the gastric antrum, confirming the suspected diagnosis of BBS.

A $2.5 \mathrm{~mm}$ ball-tip, needle-type knife was initially used to incise the granulomatous tissue, allowing intra-gastric passage of a guidewire, pushed through the cutaneous aspect of the PEG tract. The use of a novel, sphincterotome-like, dedicated device, designed for radial incision of BBS-related intra-gastric granulomatous tissue (Flamingo Set, Medwork, Höchstadt, Germany) was then applied. This device was inserted over the guidewire into the stomach, through the external aspect of the partially cut PEG tube. The guidewire was subsequently withdrawn and the distal part of the Flamingo device was flexed by $180^{\circ}$, exposing the bow-string, sphincterotome-like, cutting wire. External traction was then applied to the Flamingo device from the cutaneous side of the PEG tract. Optimal apposition of the cutting wire and the granulomatous tissue was achieved through direct endoscopic visualisation. The overgrown tissue was then incised by a series of radial cuts until the plastic bumper was exposed. The PEG bumper and remnant of the externally cut PEG tube was then released into the gastric lumen through gentle, external manipulation. As a pre-cautionary measure, the excision site was partially closed by deployment of through-the-scope endoclips. The whole procedure was performed under conscious sedation and broad-spectrum, intravenous antibiotic prophylaxis; no immediate, early or late adverse events were encountered. A new PEG insertion was successfully achieved at an alternative site, 2 weeks later.

Conclusions To the best of our knowledge, this is the first use of the 'Flamingo Set' for BBS. Through our preliminary experience, this novel, dedicated device appears to be user-friendly, safe, quick and effective for minimally invasive, endoscopic management of BBS and warrants further study.

\section{PTH-063 SUSPICION OF DEEP SUBMUCOSAL INVASION DURING ENDOSCOPIC SUBMUCOSAL DISSECTION: SIGNIFICANCE OF THE MUSCLE-RETRACTING SIGN}

${ }^{1}$ Edward J Despott, ${ }^{1}$ Alberto Murino, ${ }^{1}$ Nikolaos Lazaridis*, ${ }^{1}$ Nikolaos Koukias, ${ }^{1}$ Andrea Telese, ${ }^{1}$ Deborah Costa, ${ }^{1}$ Claudia Coppo, ${ }^{1,2}$ Yoshikazu Hayashi, ${ }^{2}$ Hironori Yamamoto. ${ }^{1}$ The Royal Free Unit for Endoscopy, The Royal Free Hospital And University College London (UCL) Institute For Liver And Digestive Health, London, UK; ${ }^{2}$ Division of Gastroenterology, Department of Medicine, Jichi Medical University, Shimotsuke, Japan

\subsection{6/gutjnl-2019-BSGAbstracts.88}

Introduction Colorectal endoscopic submucosal dissection (ESD) is a well-established minimally invasive resection technique. When the so-called muscle-retracting (MR) sign is encountered during ESD, complete resection may not be feasible. The pocket creation method (PCM) allows easier recognition of the submucosal space in the context of fibrosis and MR sign. To date, both magnifying endoscopy and endoscopic ultrasound may not be able to show invasive cancer, especially for lateral spreading tumor (LST) with a large nodule. Therefore it may be difficult to predict if any MR sign is caused by fibrosis or deep submucosal invasion.

Methods Our aim was to highlight the characteristics of deep submucosal invasion during PCM-ESD. A 74-year-old man had a colonoscopy due to haematochezia and a large granular, mixed-nodular LST was identified in the proximal rectum. Endoscopic assessment of the lesion with near focus, indigo carmine and narrow band imaging (NBI) did not reveal any sign of Kudo pit pattern Vn, JNET type 3 surface findings, or any other definitive sign of intramucosal or deeply invasive cancer. For this reason we proceeded with saline-immersion therapeutic endoscopy (SITE) facilitated PCM-ESD.

Results After dissection of the distal part of the lesion, the MR sign was encountered within the submucosal pocket, underneath a large nodule. Despite continuing dissecting this severely fibrotic submucosal area using the PCM technique, increasing severity of submucosal fibrosis and repeated bleeding from convergent, irregular submucosal neovascularisation around the MR site (with an appearance akin to 'solar flares'), impeded further resection. ESD was therefore discontinued due to high suspicion for submucosal invasion. Histopathological analysis of biopsies taken from the MR area confirmed deep submucosal invasion.

Conclusions Our findings reinforce the suspicion that a flare of neovascularisation convergent onto the MR area is suggestive of deep submucosal invasion. In this scenario ESD could be discontinued and surgical options should be considered.

\section{PTH-064 SUCCESSFUL ERCP AND PERI-HILAR STENTING IN A PATIENT WITH SITUS INVERSUS - A UK FIRST}

Cameron Green, Kohilan Gananandan*, Sudeep Tanwar. Whipps Cross University Hospital, Barts Health NHS Trust, London, UK

\subsection{6/gutjnl-2019-BSGAbstracts.89}

Introduction Complete situs inversus (CSI) is a rare autosomal recessive genetic abnormality (incidence of 1 in 10000 live births) in which there is left to right transposition of all viscera and dextroversion of the heart. Herein we report the first reported case in the UK of a patient with a CSI undergoing therapeutic ERCP for choledolithiasis (Cotton Grade 3). 